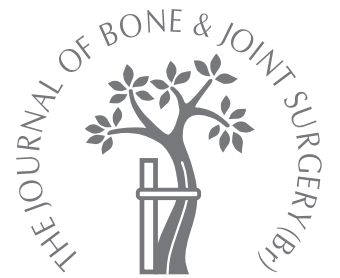

B. A. Rogers, P. Thornton-Bott, S. R. Cannon, T. W. R. Briggs

From the Royal National Orthopaedic Hospital, Stanmore, England

\title{
Interobserver variation in the measurement of patellar height after total knee arthroplasty
}

We assessed the reproducibility and accuracy of four ratios used to measure patellar height, namely the Blackburne-Peel, Caton-Deschamps, Insall-Salvati and modified Insall-Salvati, before and after total knee arthroplasty. The patellar height was measured, by means of the four ratios, on the pre- and post-operative lateral radiographs of 44 patients (45 knees) who had undergone total knee arthroplasty. Two independent observers measured the films sequentially, in identical conditions, totalling 720 measurements per observer. Statistical analysis, comparing both observers and ratios, was carried out using the intraclass correlation coefficient.

Before operation there was greater interobserver variation using either the Insall-Salvati or modified Insall-Salvati ratios than when using the Caton-Deschamps or Blackburne-Peel methods. This was because of difficulty in identifying the insertion of the patellar tendon. Before operation, there was a minimal difference in reliability between these methods. After operation the interobserver difference was greatly reduced using both the CatonDeschamps and Blackburne-Peel methods, which use the prosthetic joint line, compared with the Insall-Salvati and modified Insall-Salvati, which reference from the insertion of the patellar tendon.

The theoretical advantage of using the Insall-Salvati and modified Insall-Salvati ratios in measuring true patellar height after total knee arthroplasty needs to be balanced against their significant interobserver variability and inferior reliability when compared with other ratios.

B. A. Rogers, MA, MSc, MRCGP, MRCS, Special Registrar

Royal Surrey County Hospital, Guildford, Surrey.

P. Thornton-Bott, MRCS, Specialist Registrar John Radcliffe Hospital, Headley Way, Headington, Oxford OX3 9DZ, UK.

S. R. Cannon, FRCS, Consultant Orthopaedic Surgeon

T. W. R. Briggs, MCh(Orth), FRCS, Consultant Orthopaedic Surgeon

Royal National Orthopaedic Hospital, Brockley Hill,

Stanmore HA7 4LP, UK.

Correspondence should be sent to $\mathrm{Mr}$ B. A. Rogers at 38 Woodham Waye, Woking, Surrey GU21 5SJ, UK; e-mail: benedictrogers@hotmail.com

(C)2006 British Editorial Society of Bone and Joint Surgery doi:10.1302/0301-620X.88B4. $16407 \$ 2.00$

$J$ Bone Joint Surg [Br] 2006;88-B:484-8.

Received 16 February 2005; Accepted after revision 9 November 2005
The patella plays a crucial role in the biomechanics of the knee by extending the lever arm of the extensor mechanism, thus improving the demonstrable strength of quadriceps by between $30 \%$ and $50 \% .{ }^{1,2}$ The articulation of the patella within the femoral condylar groove creates a joint reaction force which relates both to the degree of knee flexion and contraction of quadriceps. This force in full flexion of the knee when load-bearing can approach five to seven times the body-weight. ${ }^{3}$

The height of the patella alters the joint reaction force for any particular point in the flexion-extension cycle of the knee. A high riding patella, patella alta, may result in chondromalacia patellae, tendonitis of both the patellar and quadriceps tendons, and patellofemoral instability. ${ }^{4-6}$ A low riding patella, patella baja or infera, may be developmental (patella infera syndrome), or because of trauma, neurological disorders, or may occur after surgery on the knee. Limitation of movement, OsgoodSchlatter disease and patellofemoral arthritis may all result from patella baja. ${ }^{7}$ Patello- femoral symptoms are responsible for a large percentage of revisions of total knee arthroplasty (TKA). ${ }^{8-11}$

Because the femoral condylar groove is difficult to define accurately radiologically, several ratios for the measurement of patellar height have been developed which relate the patella to the proximal tibia, namely the BlackburnePeel, ${ }^{12}$ Caton-Deschamps, ${ }^{13}$ Insall-Salvati ${ }^{6}$ and modified Insall-Salvati methods. ${ }^{14}$

There are few studies on the interobserver variation of the measurement of patellar height. Berg, Mason and Lucas ${ }^{15}$ studied 15 patients with three observers and showed that the Blackburne-Peel method was relatively reproducible. Seil et al ${ }^{16}$ also showed that this method had the lowest interobserver variability when assessing patellar height, in a study of 21 patients with symptomatic knees. However, Aparicio et $\mathrm{al}^{17}$ studied lateral radiographs of the knee in 36 children and found that the Caton-Deschamps ratio was more reliable and reproducible than the BlackburnePeel. 


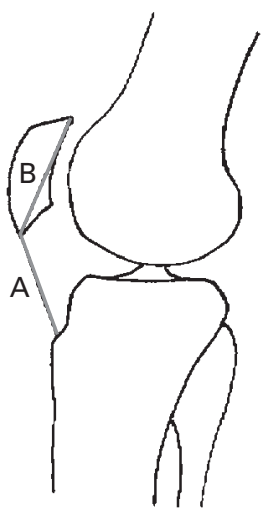

Pre-operative

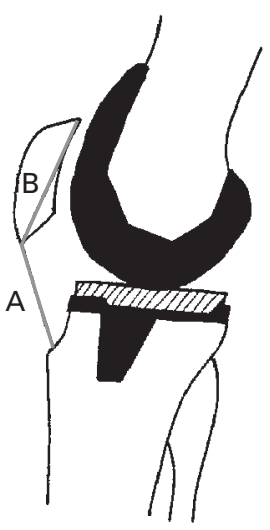

Post-operative

Fig. 1a

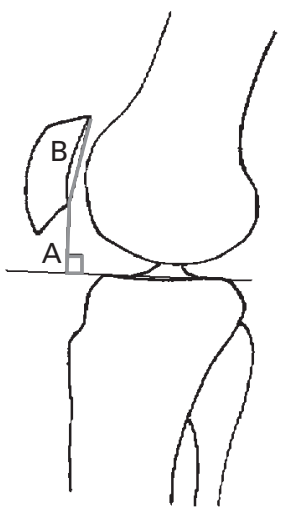

Pre-operative

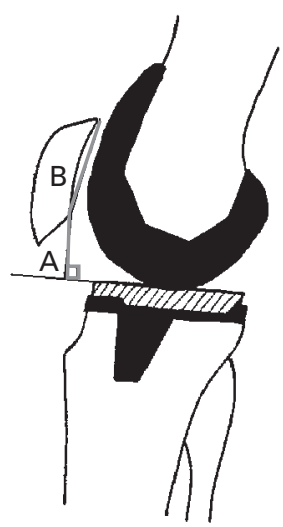

Post-operative

Fig. 1c

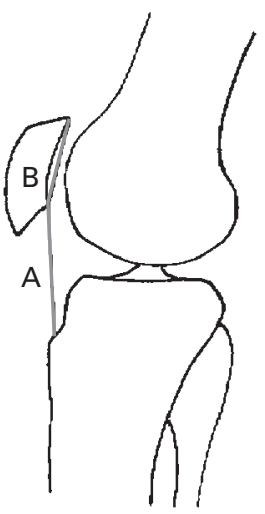

Pre-operative

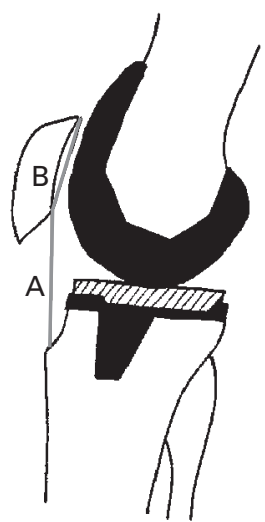

Post-operative

Fig. 1b

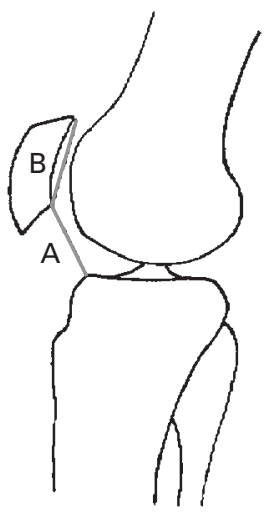

Pre-operative

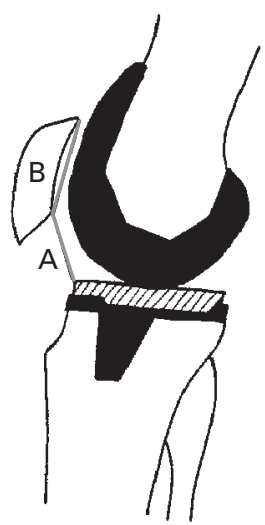

Post-operative

Fig. 1d

Diagrams showing the four methods of measuring patellar height. Figure 1a - The Insall-Salvati ratio: A, the initial flare/notch of the anterior proximal tibia to the inferior patellar pole; B, inferior pole to superior pole of patella. Figure $1 \mathrm{~b}-\mathrm{The}$ modified InsallSalvati ratio: $A$, the initial flare/notch of the anterior proximal tibia to the inferior aspect of the patellar articular surface; $B$, length of the patellar articular surface. Figure 1c-The Blackburne-Peel ratio: A, the perpendicular height from the tibial articular surface to the inferior aspect of the patellar articular surface; $B$, the length of the patellar articular surface. Figure 1d - The CatonDeschamps ratio: $A$, the anterior border of the tibial plateau to the inferior aspect of the patellar articular surface; $B$, the length of the patellar articular surface.

Scuderi, Windsor and Insall ${ }^{18}$ showed differences in the incidence of patella baja after high tibial osteotomy $(89 \%$ vs $73 \%$ ) depending on whether the Insall-Salvati or Blackburne-Peel ratio was used. After TKA, Koshino et al ${ }^{19}$ found a significant incidence of patella baja when measured using the Insall-Salvati ratio. It has been proposed that neither the Blackburne-Peel nor Caton-Deschamps ratio should be used to diagnose patella baja after TKA since they are altered by the position of the joint line. ${ }^{5}$

By using these four ratios, we have assessed the reliability and interobserver variability in the measurement of the patellar height for patients who have undergone TKA.

\section{Patients and Methods}

The lateral radiographs of the knee of 44 patients who had undergone a Kinemax TKA (Stryker, Newbury, United Kingdom) were evaluated. The operations had been per- formed at our institution, with osteoarthritis or rheumatoid arthritis being the only indications. One patient had undergone bilateral TKA and the radiographs from both procedures, which were performed at different times, were included. Patients who had undergone a high tibial osteotomy, or a revision procedure, were excluded from the study.

Lateral radiographs were taken before and after the operation with the knee in at least $20^{\circ}$ of flexion. The patellar height was measured manually by two of the authors (BAR, PT-B) in an independent sequential manner, and under identical conditions. Each examiner was blinded to the patients' outcome or the conclusions of the other examiner.

Each ratio was derived from two measurements, one below (measurement $\mathrm{A}$ ) and one above the lower patella (measurement B). Four main methods of measuring patellar 
Table I. Individual and overall ratios for the four methods used, before and after total knee arthroplasty

\begin{tabular}{|c|c|c|c|c|c|c|c|c|}
\hline & \multicolumn{4}{|c|}{ Pre-operative ${ }^{*}$} & \multicolumn{4}{|c|}{ Post-operative } \\
\hline & CD & BP & IS & mod IS & CD & BP & IS & $\bmod I S$ \\
\hline \multicolumn{9}{|l|}{ Measurement A } \\
\hline Interobserver difference & 2.73 & 2.07 & 4.50 & 4.79 & 1.36 & 1.60 & 4.62 & 4.79 \\
\hline $\mathrm{ICC}^{\dagger}$ & 0.67 & 0.54 & 0.61 & 0.66 & 0.85 & 0.87 & 0.52 & 0.53 \\
\hline $95 \% \mathrm{Cl}$ & 0.52 to 0.74 & 0.42 to 0.66 & 0.47 to 0.81 & 0.45 to 0.81 & 0.75 to 0.95 & 0.78 to 0.93 & 0.30 to 0.73 & 0.27 to 0.73 \\
\hline \multicolumn{9}{|l|}{ Measurement B } \\
\hline Interobserver difference & 0.67 & 0.67 & 1.19 & 0.67 & 1.05 & 1.05 & 1.14 & 1.05 \\
\hline ICC & 0.80 & 0.80 & 0.93 & 0.80 & 0.84 & 0.84 & 0.58 & 0.84 \\
\hline $95 \% \mathrm{Cl}$ & 0.65 to 0.88 & 0.65 to 0.88 & 0.87 to 0.96 & 0.65 to 0.88 & 0.56 to 0.86 & 0.56 to 0.86 & 0.45 to 0.65 & 0.56 to 0.86 \\
\hline \multicolumn{9}{|l|}{ Overall } \\
\hline Interobserver difference & 0.10 & 0.08 & 0.11 & 0.17 & 0.05 & 0.05 & 0.11 & 0.16 \\
\hline ICC & 0.53 & 0.62 & 0.58 & 0.54 & 0.82 & 0.83 & 0.52 & 0.48 \\
\hline $95 \% \mathrm{Cl}$ & 0.45 to 0.63 & 0.49 to 0.67 & 0.44 to 0.65 & 0.47 to 0.59 & 0.69 to 0.95 & 0.71 to 0.90 & 0.49 to 0.60 & 0.36 to 0.56 \\
\hline
\end{tabular}

* CD, Caton-Deschamps; BP, Blackburne-Peel; IS, Insall-Salvati; mod IS, modified Insall-Salvati

$\dagger$ ICC, intraclass correlation co-efficient

¥ 95\% Cl, 95\% confidence interval

height were recorded for all the radiographs (Fig. 1), with a total of 720 measurements (360 ratios) per examiner. Measurement A was taken from the point of insertion of the patellar tendon on the anterior aspect of the proximal tibia to the lower pole of the patella for the Insall-Salvati ratio and to the inferior aspect of the articular surface of the patella for the modified Insall-Salvati ratio. In radiographs in which the patellar tendon was not readily identifiable, measurement A was taken from the position of the initial flare or notch in the proximal part of the anterior aspect of the tibia.

For the modified Insall-Salvati, Caton-Deschamps and Blackburne-Peel measurements, measurement $\mathrm{B}$ was the length of the articular surface of the patella excluding osteophytes. For the Caton-Deschamps ratio, measurement A was the distance from the inferior point of measurement $B$ to the most superior point of the anterior surface of the tibia, whereas for the Blackburne-Peel ratio it was the perpendicular distance between the inferior point of measurement $B$ and a line drawn tangentially from the tibial surface (Fig. 1). The post-operative measurement of $\mathrm{A}$ in both the Caton-Deschamps and Blackburne-Peel ratios was from the anterosuperior corner of the polyethylene insert (Fig. 1), a point readily identifiable on lateral radiographs.

An adjustment of $7 \mathrm{~mm}$ was added to measurement $\mathrm{A}$ for the post-operative Blackburne-Peel measurement ratio in order to account for the mean depth $(5.8 \mathrm{~mm}$ to $7.5 \mathrm{~mm})$ of the polyethylene insert, thus ensuring that this measurement related to the joint line. This measurement was specific to the Kinemax insert and is likely to be different in other types of TKA.

Statistical analysis. The data were collated on Excel (Microsoft, Redmond, Washington) with comparisons subsequently made between ratios for both pre- and post-operative radiographs, interobserver variability and reliability. Intraclass correlation coefficient statistical analysis was undertaken using SPSS version 12 (SPSS Inc., Chicago, Illinois).

\section{Results}

The mean interobserver variation and intraclass correlation co-efficient, with $95 \%$ confidence intervals (CI), before and after TKA for measurements $\mathrm{A}$ and $\mathrm{B}$, and the overall ratio for each of the four methods, are shown in Table I. Preoperatively, there was less interobserver variation and greater reliability with measurement B compared with measurement A for all methods.

The post-operative intraclass correlation co-efficient for measurement A improved relative to its pre-operative value when using the Caton-Deschamps (0.67 to 0.85) and Blackburne-Peel (0.54 to 0.87$)$ ratios, but deteriorated using the Insall-Salvati (0.61 to 0.52 ) and modified Insall-Salvati (0.66 to 0.53 ) ratios. There was, therefore, poor reproducibility for measurement A post-operatively using the InsallSalvati and modified Insall-Salvati ratios. Apart from the Blackburne-Peel ratio, there were small improvements in the post-operative correlation of measurement B. The resulting post-operative intraclass correlation coefficient between ratios showed greater reproducibility using the Caton-Deschamps and Blackburne-Peel methods $(0.82$ and 0.83 ) compared with that using the Insall-Salvati and modified Insall-Salvati ratios (0.52 and 0.48).

Overall, there was an improvement in the post-operative ratio compared with pre-operatively when using the CatonDeschamps (0.53 to 0.82) and Blackburne-Peel (0.62 and $0.83)$ methods. There were small reductions in the postoperative correlation for both the Insall-Salvati and modified Insall-Salvati ratios.

The results indicate that interobserver variation in the post-operative measurement $A$ is principally responsible for the deterioration of reproducibility of the Insall-Salvati and modified Insall-Salvati ratios. In both these ratios, measurement A represented the length of the deep surface of the patellar tendon. In the original measurements of Insall, he stated that if the patellar tendon could not be adequately visualised, its length could be gauged by using a "clearly defined notch" on the anterior aspect of the proximal tibia 


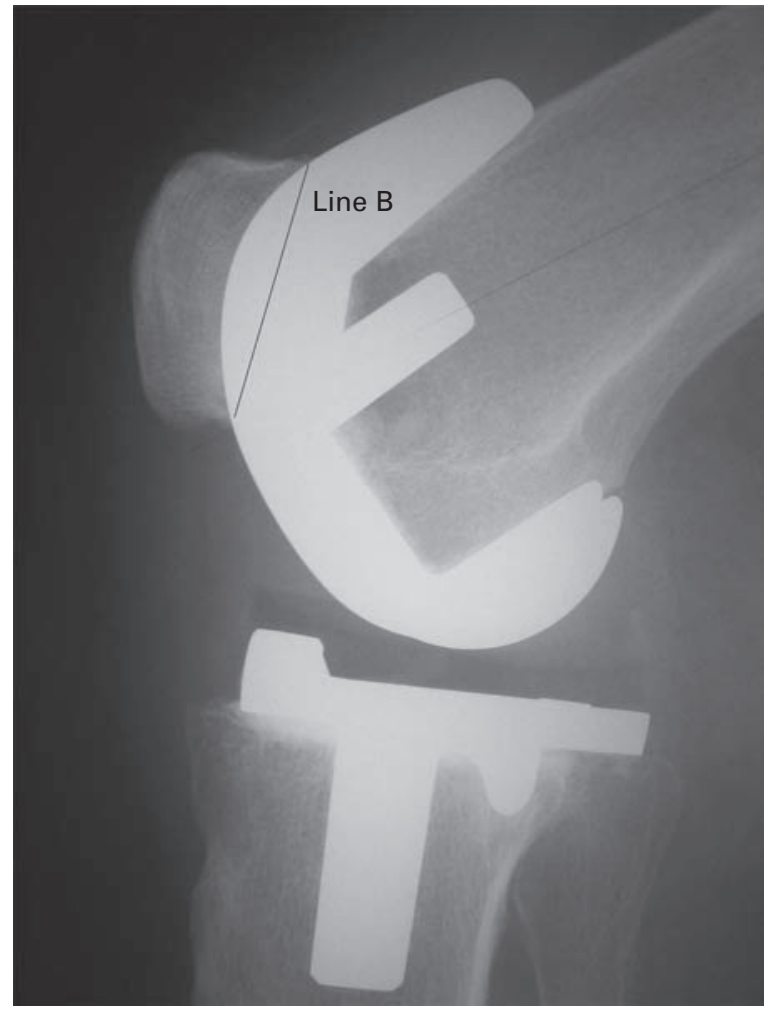

Fig. 2

Radiograph showing line B which is the estimated length of the articular surface of the patella.

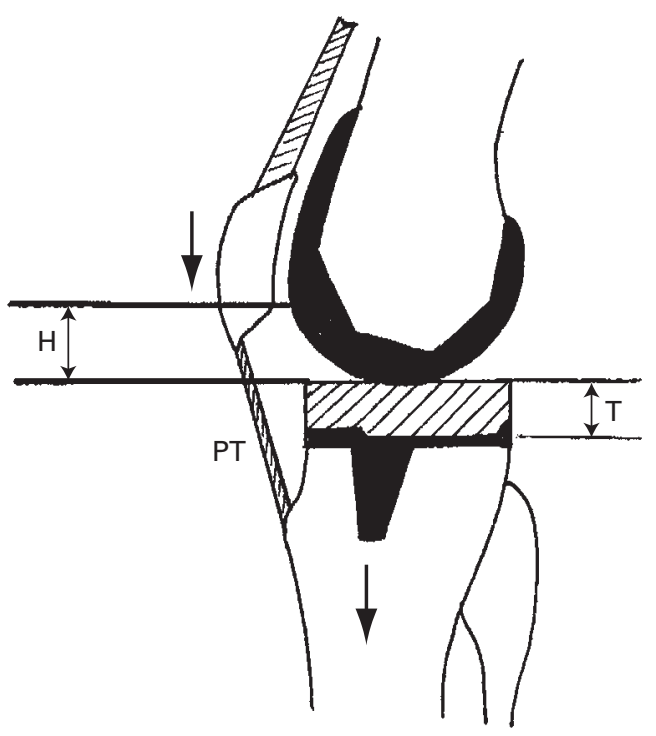

Fig. 3

Diagram showing the effect of the thickness of the tibial insert on the position of the patella. As the thickness $T$ of the insert increases, the position of the tibia becomes more distal. The inelastic patellar tendon (PT), attached to the displaced tibia, pulls the patella distally thus reducing the patellar height $\mathrm{H}$. as a point of reference. ${ }^{3}$ However, we had difficulty in identifying such a notch or flare in ready radiographs, with the presence of multiple notches or a smooth convex proximal tibial profile being common problems.

There are inaccuracies inherent in determining measurement B with the Blackburne-Peel, Caton-Deschamps and modified Insall-Salvati ratios, since it represents the length of the articular surface of the patella. Before TKA, provided that osteophytes were ignored, this was relatively uncomplicated. However, the articular surface of the patella may not be fully visible after replacement arthroplasty since it may be partially located within the trochlear groove of the femur (Fig. 2) and its length must be estimated.

\section{Discussion}

The results from our study indicate that, for patients undergoing TKA, there are significant differences in reliability and interobserver variability in the four main methods used to measure patellar height. These methods rely on the positional relationship between the patella and proximal tibia, while the insertion of the prosthesis alters the accuracy and reproducibility. These differences also vary depending on whether patellar height is measured before or after TKA.

The Blackburne-Peel and Caton-Deschamps ratios both require the precise identification of the proximal joint surface of the tibia for their evaluation. In joints with a significant amount of osteoarthritis or rheumatoid arthritis, visualisation of this surface is difficult and may need to be estimated. However, after TKA the tibial insert provides a precise point of reference, thereby improving the interobserver variability for these methods (Fig. 1). Our study, in order to give an accurate measurement to the new joint surface, compensated for the dishing of the tibial insert in the determination of measurement A for the Blackburne-Peel ratio. A similar compensation is difficult to introduce into the Caton-Deschamps measurement, since unlike the Blackburne-Peel method, it is not made perpendicular to the joint surface.

The application of a particular ratio to the measurement of patellar height in TKA depends on the information required by the clinician. The original description of patella baja was defined before joint replacement surgery and was related to shortening of the patellar tendon, distal positioning of the patella relative to the femoral trochlea and a reduction of the distance between the patella and tibial surface. After TKA, however, the patella may be positioned distal to the femoral condyles and closer to the joint surface of the tibia while the patellar tendon remains a constant length. This has been termed pseudo-patella baja, a reduction in patellar height relative to the joint surface ${ }^{5}$ and is related to the thickness of the insert (Fig. 3). It can be due to 'overstuffing' of the knee, or as a necessary consequence of softtissue release and occurs when the thickness of the tibial tray plus insert is greater than the thickness of tibia removed.

True patella baja necessitates shortening of the patellar tendon, so its measurement requires indices which relate to 
the tibial tuberosity and not to either the tibial plateau or the tibial component of a TKA. The Insall-Salvati and modified Insall-Salvati ratios relate the length of the patella to the length of the patellar tendon and are therefore independent of the joint surface.

The biomechanics of the patellofemoral joint are related to the position of the patella within the trochlear groove and changes to this relationship have been shown to be detrimental. During weight-bearing, the position of the femoral condyles, and hence the trochlear groove, is directly related to the position of the joint line. It is therefore logical to measure the height of the patella from the joint surface, as changes in this height will indicate potential problems. This is especially the case in TKA in which changes in the position of the joint line will not be identified by measuring the length of the patellar tendon.

Despite the theoretical advantages of using the Insall-Salvati and modified Insall-Salvati methods for the assessment of true patellar height, our study highlights their inferior interobserver reproducibility after TKA, mainly related to difficulties in identifying the patellar tendon and tibial landmarks on post-operative radiographs. However, although the Blackburne-Peel and Caton-Deschamps ratios show a greater degree of correlation after TKA, they are affected by the changes in the position of the joint line and do not accurately correlate with true patellar height.

Thus, for the assessment of patellar height in patients undergoing TKA, the clinician should tailor the ratio used to the requirements. Measurement of the true patellar height and identification of true patella baja or alta, necessitate the use of the Insall-Salvati or modified Insall-Salvati ratio. However, these ratios have inferior interobserver correlation and reproducibility; measurements after operation are misleading if the position of the joint line has been altered. The Blackburne-Peel and Caton-Deschamps ratios evaluate patellar height relative to the joint surface and will identify pseudo-patella baja. These methods have superior reliability and interobserver correlation after TKA.
We wish to acknowledge the valuable help and comments of $\operatorname{Dr}$ A. Saifuddin (Consultant Radiologist, Royal National Orthopaedic Hospital) in the preparation of this paper.

No other benefits have been received or will be received from a commercial party related directly or indirectly to the subject of this article.

\section{References}

1. Krevolin JL, Pandy MG, Pearce JC. Moment arm of the patellar tendon in the human knee. J Biomech 2004;37:785-8.

2. Wahrenberg H, Lindbeck L, Ekholm J. Knee muscular moment, tendon tension force and EMG during a vigorous movement in man. Scand J Rehabil Med 1978;10: 99-106.

3. Mow VC, Flatow EL, Ateshian GA. Biomechanics. In: Buckwater JA, Einhorn TA, Simon SR, eds. Orthopaedic basic science. American Academy of Orthopaedic Surgeons, 2000:134-80.

4. Grana WA, Kriegshauser LA. Scientific basis of extensor mechanism disorders. Clin Sports Med 1985;4:247-57.

5. Grelsamer RP. Patella baja after total knee arthroplasty: is it really patella baja? J Arthroplasty 2002;17:66-9.

6. Insall J, Salvati E. Patella position in the normal knee joint. Radiology 1971;101: 101-4.

7. Aparicio G, Abril JC, Calvo E, Alvarez L. Radiologic study of patellar height in Osgood-Schlatter disease. J Pediatr Orthop 1997;17:63-6.

8. Bryan RS, Rand JA. Revision total knee arthroplasty. Clin Orthop 1982;170:116-22.

9. Cameron HU, Hunter GA. Failure in total knee arthroplasty: mechanisms, revisions, and results. Clin Orthop 1982;170:141-6.

10. Clayton ML, Thirupathi R. Patellar complications after total condylar arthroplasty. Clin Orthop 1982;170:152-5.

11. Mochizuki RM, Schurman DJ. Patellar complications following total knee arthroplasty. J Bone Joint Surg [Am] 1979;61-A:879-83.

12. Blackburne JS, Peel TE. A new method of measuring patellar height. J Bone Joint Surg [Br] 1977:59-B:241-2.

13. Caton J, Deschamps G, Chambat $\mathbf{P}$, et al. Patella infera: a propos of 128 cases. Rev Chir Orthop Reparatrice Appar Mot 1982;68:317-25 (in French).

14. Grelsamer RP, Meadows S. The modified Insall-Salvati ratio for assessment of patellar height. Clin Orthop 1992;282:170-6.

15. Berg EE, Mason SL, Lucas MJ. Patellar height ratios: a comparison of four measurement methods. Am J Sports Med 1996;24:218-21.

16. Seil R, Muller B, Georg T, Kohn D, Rupp S. Reliability and interobserver variability in radiological patellar height ratios. Knee Surg Sports Traumatol Arthrosc 2000;8: 231-6.

17. Aparicio G, Abril JC, Albinana J, Rodriguez-Salvanes F. Patellar height ratios in children: an interobserver study of three methods. J Pediatr Orthop B 1999;8:29-32.

18. Scuderi GR, Windsor RE, Insall JN. Observations on patellar height after proximal tibial osteotomy. J Bone Joint Surg [Am] 1989;71-A:245-8.

19. Koshino T, Ejima M, Okamoto R, Morii T. Gradual low riding of the patella during postoperative course after total knee arthroplasty in osteoarthritis and rheumatoid arthritis. J Arthroplasty 1990;5:323-7. 